\title{
Locally Restricted Compositions III. Adjacent-Part Periodic Inequalities
}

\author{
Edward A. Bender \\ Department of Mathematics \\ University of California, San Diego \\ La Jolla, CA 92093-0112 \\ ebender@ucsd.edu
}

\author{
E. Rodney Canfield* \\ Department of Computer Science \\ University of Georgia \\ Athens, GA 30602 \\ erc@cs.uga.edu
}

Submitted: Aug 26, 2010; Accepted: Oct 19, 2010; Published: Oct 29, 2010

Mathematics Subject Classifications: 05A15, 05A16

\begin{abstract}
We study compositions $c_{1}, \ldots, c_{k}$ of the integer $n$ in which adjacent parts may be constrained to satisfy some periodic inequalities, for example

$$
c_{2 i}>c_{2 i+1}<c_{2 i+2} \quad \text { (alternating compositions). }
$$

The types of inequalities considered are $<, \leqslant,>, \geqslant$ and $\neq$. We show how to obtain generating functions from which various pieces of asymptotic information can be computed. There are asymptotically $A r^{-n}$ compositions of $n$. In a random uniformly selected composition of $n$, the largest part and number of distinct parts are almost surely asymptotic to $\log _{1 / r}(n)$. The length of the longest run is almost surely asymptotic to $C \log _{1 / r}(n)$ where $\mathrm{C}$ is an easily determined rational number. Many other counts are asymptotically normally distributed. We present some numerical results for the various types of alternating compositions.
\end{abstract}

\section{Introduction}

Locally restricted compositions $c_{1}, \ldots, c_{k}$ are compositions in which the values allowed for the part $c_{i}$ depend on the parts in a fixed width window ending at $c_{i}$ as well as on the value of $i$ modulo some modulus $m$. The earliest locally restricted compositions to be studied were Carlitz compositions which require adjacent parts to differ. In [2] we generalized Carlitz compositions to the case in which the difference between adjacent parts must lie in some (nearly arbitrary) set of integers $\mathcal{D}$, obtaining generating functions and a variety of information. (For Carlitz compositions, $\mathcal{D}$ is the set of nonzero integers.) In this paper

\footnotetext{
*Research supported by NSA Mathematical Sciences Program.
} 
we study a different generalization of Carlitz compositions: Here we require adjacent parts to satisfy an inequality, be unequal, or have no restriction; and the condition depends on the position of the part modulo some $m$. Carlitz compositions have $m=1$ and $c_{i} \neq c_{i+1}$. Strictly alternating compositions have $m=2$ and $c_{i}<c_{i+1}>c_{i+2}$.

In [3] we studied the general case of locally restricted compositions, obtaining various results. For convenience, we state these results in Theorem 1 below for the classes of compositions considered in this paper. Unfortunately [3] provides no method for computing reasonably accurate numerical information. The main goal of this paper is to discuss a method for obtaining generating functions from which some of the parameters in Theorem 1 can be estimated, and to apply the method to alternating compositions.

A common framework for this paper and [2] would require $c_{i+1}-c_{i} \in \mathcal{D}_{i}$, where the subscript on $\mathcal{D}_{i}$ is interpreted modulo $m$. Of course [3] contains such a generalization, but it does not provide generating functions or numerical values. We have been unable to find an approach that does.

In this paper, relations between adjacent parts take one of the six forms

$$
a_{i-1}<a_{i}, \quad a_{i-1} \leqslant a_{i}, \quad a_{i-1}>a_{i}, \quad a_{i-1} \geqslant a_{i}, \quad a_{i-1} \neq a_{i} \quad \text { and unrestricted. }
$$

Parts of size zero may or may not be allowed. These conditions are then put together in a repeating pattern; for example, $a_{4 i} \neq a_{4 i+1}<a_{4 i+2} \geqslant a_{4 i+3} \geqslant a_{4 i+4}$. There are two requirements for the conditions:

\section{Either}

(a) "unrestricted" must appear or

(b) "not equal" $\left(c_{i-1} \neq c_{i}\right)$ must appear or

(c) both increasing and decreasing inequalities must appear.

If this were not the case, we would be dealing with partitions, which behave differently from other restricted compositions.

2. If none of $<,>$ and $\neq$ appear, then parts of size zero are not allowed. Without this restriction, $n$ would have infinitely many compositions since a composition could have arbitrarily many adjacent zero parts.

Recurrent local properties. A local property (of width $w$ ) is a property that holds for the sequence of parts $c_{i}, c_{i+1}, \ldots, c_{i+w-1}$. It may include restrictions on $i$ modulo $m$. For example, " $c_{i}=3$ " is a local property for any width $w \geqslant 1$ and " $c_{2 i}+c_{2 i+2}$ is odd" is a local property for any width $w \geqslant 3$. On the other hand, "the next occurrence of a part equal to $c_{i}$ is an odd distance from $c_{i}$ " is not a local property. A local property is recurrent if it can occur almost arbitrarily often. More specifically, there is a $j$ such that for each $k$ there is an $n$ such that for $1 \leqslant i \leqslant k$, there is a composition of $n$ in which the number of occurrences of the property is between $(i-1) j$ and $i j$. The examples given earlier in this paragraph are recurrent for the compositions we are considering.

Runs. A run is a sequence of parts that is repeated. Thus 2, 1, 3, 1, 3, 1, 4, 2, 2, 3 contains the maximal runs $1,3,1,3$ and $3,1,3,1$ and 2,2 . The length of a run is the number of parts it contains. 
Theorem 1 The following properties of compositions with periodic adjacent-part inequality constraints follow from the general case in [3]. After each property we give a parenthetic reference to the relevant part of that paper. When randomness is mentioned, compositions of $n$ are sampled uniformly. All asymptotics are as $n \rightarrow \infty$.

(a) The number of compositions of $n$ is asymptotic to $A r^{-n}$ with an exponentially small relative error. (Theorem 3)

(b) Suppose the index in the constraints is shifted, for example, with alternating compositions we may replace $a_{2 i}<a_{2 i+1}>a_{2 i+2}$ with $a_{2 i+1}<a_{2 i+2}>a_{2 i+3}$. And/or suppose we do or do not require that the number of parts be a multiple of the period. The value of $A$ in (a) will change, but the value of $r$ and the base of the exponential in the relative error does not. (Example 4)

(c) The number of distinct parts and the largest part are each almost surely asymptotic to $\log _{1 / r}(n)$. (Section 9)

(d) Suppose arbitrarily long runs can be based on repetitions of $\overrightarrow{\mathbf{p}}$. The number of parts in the longest run based on $\overrightarrow{\mathbf{p}}$ is almost surely asymptotic to $(1 / \mu(\overrightarrow{\mathbf{p}})) \log _{1 / r}(n)$, where $\mu(\overrightarrow{\mathbf{p}})$ is the average size of parts in $\overrightarrow{\mathbf{p}}$. (Theorem 5)

(e) Let the random variable $X_{n}$ be the number of occurrences of recurrent local property. The distribution of $X_{n}$ is asymptotically normal with mean and variance asymptotic to $\mu$ and $\sigma^{2} n$ for some positive $\mu$ and $\sigma$. (Theorem 4)

One can easily deduce other, perhaps less interesting, properties from the general formulation in [3]. For example, the proof of Theorem 4 in that paper is easily adapted to show that the conclusion in (e) holds for random variables such as "the sum of parts exceeding 3" and "the sum of parts $c_{2 i+1}$."

Example 1 (Run lengths) When all the conditions are weak inequalities, it follows from (e) that the longest run almost surely consists of repetitions of 1 and has length asymptotic to $\log _{1 / r}(n)$. Suppose we are dealing with alternating compositions containing at least one strong inequality. If 0 is allowed as a part, then the longest run is almost surely an alternating sequence of 0 and 1 with a length asymptotic to $2 \log _{1 / r}(n)$. If 0 is not allowed as a part, then the longest run is almost surely an alternating sequence of 1 and 2 with a length asymptotic to $(2 / 3) \log _{1 / r}(n)$.

Estimating $A$ and $r$ from generating functions is practical for small $m$. For most recurrent local conditions, we are unable to obtain generating functions and so estimating $\mu$ and $\sigma$ in (e) appears difficult in most cases. An exception is the number of parts, which counts the recurrent local condition " $c_{i}$ is a part". We can also keep track of the last part and so obtain information about its distribution, which is not asymptotically normal. All this is discussed in Sections 2 and 3. In Section 4 we specialize to alternating compositions and present some numerical results showing the rapid convergence of the asymptotic estimates. If you are interested only in results for alternating compositions, skip to Section 4. 


\section{Obtaining Generating Functions}

We build up generating functions by adding one part at a time. As a result, we need to keep track of both the sum of parts and the size of the last part. We can easily keep track of the number of parts. If the period of the constraints is $m$, we obtain $m$ generating functions, $f_{i}(x, t, z)$ where $i$ keeps track of the number of parts modulo $m, x$ keeps track of the sum of parts, $t$ keeps track of the number of parts and $z$ keeps track of the size of last part. By appending a new last part to compositions counted by $f_{i-1}$, we obtain an equation for $f_{i}$. Thus we obtain a system of $m$ functional equations. With one exception to be noted shortly, here they are:

$$
\begin{aligned}
\text { if } a_{i-1} \leqslant a_{i}, & f_{i}(x, t, z) & =\frac{f_{i-1}(x, t, x z)}{1-x z} t \\
\text { if } a_{i-1}<a_{i}, & f_{i}(x, t, z) & =\frac{x z f_{i-1}(x, t, x z)}{1-x z} t \\
\text { if } a_{i-1} \geqslant a_{i}, & f_{i}(x, t, z) & =\frac{(x z)^{\delta} f_{i-1}(x, t, 1)-x z f_{i-1}(x, t, x z)}{1-x z} t ; \\
\text { if } a_{i-1}>a_{i}, & f_{i}(x, t, z) & =\frac{(x z)^{\delta} f_{i-1}(x, t, 1)-f_{i-1}(x, t, x z)}{1-x z} t \\
\text { if } a_{i-1} \neq a_{i}, & f_{i}(x, t, z) & =\frac{(x z)^{\delta} f_{i-1}(x, t, 1)}{1-x z} t-f_{i-1}(x, t, x z) t \\
\text { if unrestricted, }, & f_{i}(x, t, z) & =\frac{(x z)^{\delta} f_{i-1}(x, t, 1)}{1-x z} t
\end{aligned}
$$

where

$$
\delta= \begin{cases}0 & \text { if parts of size zero are allowed, } \\ 1 & \text { otherwise. }\end{cases}
$$

The exception to the above equations is that the equation for $f_{1}(x, z)$ must have the starting value $\frac{(x z)^{\delta} t}{1-x z}$ added.

We derive the recursion for $a_{i-1}>a_{i}$. The other derivations are similar. With $\left[z^{k}\right]$ denoting the coefficient of $z^{k}$,

$$
\begin{aligned}
f_{i}(x, t, z) & =t \sum_{k} \sum_{p=\delta}^{k-1}(x z)^{p}\left[z^{k}\right] f_{i-1}(x, t, z) \\
& =t \sum_{k} \frac{(x z)^{\delta}-(x z)^{k}}{1-x z}\left[z^{k}\right] f_{i-1}(x, t, z) \\
& =\frac{(x z)^{\delta} f_{i-1}(x, t, 1)-f_{i-1}(x, t, x z)}{1-x z} t .
\end{aligned}
$$

Example 2 (Using the recursions) To illustrate the usage of the recursions to obtain generating functions, consider the period-three constraints

$$
a_{3 i}<a_{3 i+1}>a_{3 i+2} \leqslant a_{3 i+3} \text { with no zero parts allowed. }
$$


We have

$$
\begin{aligned}
a_{3 i}<a_{3 i+1} & f_{1}(x, t, z)=\frac{x z f_{3}(x, t, x z)}{1-x z} t+\frac{t x z}{1-x z} \\
a_{3 i+1}>a_{3 i+2} & f_{2}(x, t, z)=\frac{x z f_{1}(x, t, 1)-f_{1}(x, t, x z)}{1-x z} t \\
a_{3 i+2} \leqslant a_{3 i+3} & f_{3}(x, t, z)=\frac{f_{2}(x, t, x z)}{1-x z} t .
\end{aligned}
$$

Substituting we obtain

$$
\begin{aligned}
f_{1}(x, t, z) & =\frac{t x z}{1-x z}+\frac{t^{2} x z}{1-x z} \frac{f_{2}\left(x, t, x^{2} z\right)}{1-x^{2} z} \\
& =\frac{t x z}{1-x z}+\frac{t^{3} x z}{1-x z} \frac{x^{3} z f_{1}(x, t, 1)-f_{1}\left(x, t, x^{3} z\right)}{\left(1-x^{2} z\right)\left(1-x^{3} z\right)} .
\end{aligned}
$$

Iterating this equation we obtain

$$
\begin{aligned}
f_{1}(x, t, z) & =A(x, t, z)+B(x, t, z) f_{1}(x, t, 1) \\
\text { where } A(x, t, z) & =\sum_{k=0}^{\infty} \frac{(-1)^{k} t x z}{1-x z} \prod_{i=1}^{k} \frac{t^{3} x^{3 i+1} z}{\left(1-x^{3 i-1} z\right)\left(1-x^{3 i} z\right)\left(1-x^{3 i+1} z\right)} \\
\text { and } B(x, t, z) & =\sum_{k=1}^{\infty}(-1)^{k-1} x^{3 k} z \prod_{i=1}^{k} \frac{t^{3} x^{3 i-2} z}{\left(1-x^{3 i-2} z\right)\left(1-x^{3 i-1} z\right)\left(1-x^{3 i} z\right)}
\end{aligned}
$$

With $z=1$ we have

$$
f_{1}(x, t, 1)=\frac{A(x, t, 1)}{1-B(x, t, 1)}
$$

and so

$$
f_{1}(x, t, z)=A(x, t, z)+B(x, t, z) \frac{A(x, t, 1)}{1-B(x, t, 1)} .
$$

Combining this with (3) and (4) we obtain

$$
\begin{aligned}
f_{2}(x, t, z) & =\frac{-t A(x, t, x z)}{1-x z}-\frac{B(x, t, x z) t-t x z}{1-x z} \frac{A(x, t, 1)}{1-B(x, t, 1)} \\
f_{3}(x, t, z) & =\frac{-t^{2} A\left(x, t, x^{2} z\right)}{(1-x z)\left(1-x^{2} z\right)}-\frac{B\left(x, t, x^{2} z\right) t^{2}-t^{2} x^{2} z}{(1-x z)\left(1-x^{2} z\right)} \frac{A(x, t, 1)}{1-B(x, t, 1)}
\end{aligned}
$$

If we don't care where in the period the compositions start or end, we add $\frac{t x z}{1-x z}$ to (3) and (4) as well as (2) to allow arbitrary starts and we compute

$$
f_{1}(x, t, z)+f_{2}(x, t, z)+f_{3}(x, t, z)-\frac{2 t x z}{1-x z}-t^{2} \sum_{0<i<j} x^{i}(x z)^{j}
$$

to allow arbitrary ends. (The first subtraction arises because single part compositions are counted thrice and the second arises because two part compositions with $c_{1}=i<j=c_{2}$ are counted twice.) 
Example 3 (Reverse of previous example) In the previous example, only one $f_{i}(x, t, 1)$ appeared, namely $f_{1}(x, t, 1)$ in $(3)$. If there is more than one descent, there will be more than one such $f_{i}$. To illustrate, we reverse left and right in the previous example:

$$
a_{3 i} \geqslant a_{3 i+1}<a_{3 i+2}>a_{3 i+3} \text { with no zero parts allowed. }
$$

For simplicity, we do not keep track of the number of parts. Our recursions are

$$
\begin{aligned}
a_{3 i} \geqslant a_{3 i+1} & f_{1}(x, z) & =\frac{x z f_{3}(x, 1)-x z f_{3}(x, x z)}{1-x z} \\
a_{3 i+1}<a_{3 i+2} & f_{2}(x, z) & =\frac{x z f_{1}(x, x z)}{1-x z} \\
a_{3 i+2}>a_{3 i+3} & f_{3}(x, z) & =\frac{x z f_{2}(x, 1)-f_{2}(x, x z)}{1-x z} .
\end{aligned}
$$

As in the previous example, terms such as $\frac{x z}{1-x z}$ should be added as needed. Iterating as in the previous example, we obtain an equation of the form

$$
f_{1}(x, z)=A(x, z) f_{1}(x, x)+B(x, z) f_{1}\left(x, x^{2}\right)+C(x, z),
$$

where $A, B$ and $C$ are known. Setting $z=x$ and $z=x^{2}$ gives two equations in the unknowns $f_{1}(x, x)$ and $f_{1}\left(x, x^{2}\right)$, which can be solved and substituted back into (10) to obtain an equation of the form

$$
f_{1}(x, z)=\frac{D(x, z)}{(A(x, x)-1) B(x, x)-\left(B\left(x, x^{2}\right)-1\right) A\left(x, x^{2}\right)}+R(x, z)
$$

in place of (9).

\section{Obtaining Numerical Values}

Ultimately, one obtains a generating function of the form

$$
f(x, t, z)=\frac{N(x, t, z)}{D(x, t)}+R(x, t, z) .
$$

where $D(x, 1)$ has a simple zero at $r>0$ and no other zeroes in $[0, r)$, and $N(x, 1,1)$ and $R(x, 1,1)$ have radii of convergence larger than $r$. The property of $R$ follows by computation in particular cases. The rest is due to the fact, proved in [3], that $f(x, 1,1)$ has a simple pole at $r$ and no other singularities on its circle of convergence.

Since $D(x, 1)$ can be computed to any desired accuracy and the zero is simple, one can estimate $r$ to any desired accuracy.

The value of $A$ in $\left[x^{n}\right] f(x, 1,1) \sim A r^{-n}$ is more involved. By standard results, it is $-N(r, 1,1) / r D^{\prime}(r, 1)$. 
The distribution of the last part has probability density function asymptotic to

$$
\lim _{n \rightarrow \infty} \frac{\left[x^{n}\right] N(x, 1, z)}{\left[x^{n}\right] N(x, 1,1)}=\lim _{n \rightarrow \infty} \frac{\left[x^{n}\right] N(x, 1, z)}{A r^{-n}} .
$$

Thus asymptotic moments and probabilities for given last part size are obtainable. However, the full asymptotic distribution may be difficult to obtain.

The number of parts in a random composition is asymptotically normal with mean and variance asymptotic to $\mu n$ and $\sigma^{2} n$, respectively. These require the computation of first and second partials and estimating the coefficient of $x^{n}$ in the resulting series. See [1] for further information. In particular,

$$
\mu=\frac{\partial D(r, 1,1) / \partial t}{r \partial D(r, 1,1) / \partial x} .
$$

\section{Alternating Compositions}

In this section we present results for alternating compositions; that is, those compositions such as 1,3,2,6, 4 in which the parts alternating increase and decrease. There are three variables in the type of compositions: smallest allowed part (zero or one), strict or weak increase, and strict or weak decrease.

We use notation such as $(1<\geqslant)$ to describe such compositions. Thus $(1<\geqslant)$ means that zero parts are not allowed, increases must be strict, and decreases may be weak. Of the eight possibilities, $(0 \leqslant \geqslant)$ is forbidden because the use of zeros leads to infinitely many compositions of $n$. By reversing the order of the parts $(\delta<\geqslant)$-compositions are seen to be equivalent to $(\delta \leqslant>)$ compositions. These five cases are reduced even further by noting that adding 1 to each part gives a bijection from $(0 \rho \sigma)$-compositions to $(1 \rho \sigma)$ compositions. Finally, adding 1 to down parts converts a $(0<>)$-composition to a $(1 \leqslant \geqslant)$ composition. Thus there are only two cases to consider provided our generating function has variables to keep track of the number of parts and number of down parts:

$$
\begin{aligned}
& (0<>) \text { which gives }(1<>) \text { and }(1 \leqslant \geqslant) ; \\
& (0<\geqslant) \text { which gives }(1<\geqslant), \quad(0 \leqslant>) \text { and }(1 \leqslant>) .
\end{aligned}
$$

In what follows, we do not require that a composition start at the beginning of a period nor end at the end of a period. Table 1 gives the number of alternating compositions for $n \leqslant 10$ and Table 2 shows that the asymptotic approximation $A r^{-n}$ is quite accurate.

We now derive the generating function for $(0<>)$-compositions. The approach for $(0<\geqslant)$-compositions is nearly identical.

Let the coefficient of $s^{a} t^{b} x^{n} z^{d}$ in our generating function be the number of compositions such that

- there are $a$ down parts ( $s$ "counts" number of down parts), 
- there are $b$ parts ( $t$ "counts" number of parts),

- the parts sum to $n$ ( $x$ "counts" sum of parts),

- the last part is $d$ ( $z$ "counts" last part).

Since the period is two, we introduce two functions $f_{<}$and $f_{>}$, where the subscript indicates the type of inequality involving the newly added part - a slight change from Section 2. Thus $f_{<}$deals with the case $c_{k-1}<c_{k}$. Since we are allowing both the $c_{1}<c_{2}$ and $c_{1}>c_{2}$ compositions, $f$ 's have initial conditions. The approach in Section 2 leads to

$$
\begin{aligned}
& f_{<}(s, t, x, z)=\frac{t x z}{1-x z}+f_{>}(s, t, x, x z) \frac{t x z}{1-x z} \\
& f_{>}(s, t, x, z)=\frac{s t}{1-x z}+f_{<}(s, t, x, 1) \frac{s t}{1-x z}-f_{<}(s, t, x, x z) \frac{s t}{1-x z} .
\end{aligned}
$$

\begin{tabular}{|l|rrrrrrrrrr|}
\hline & 1 & 2 & 3 & 4 & 5 & 6 & 7 & 8 & 9 & 10 \\
\hline$(0<>)$ & 4 & 8 & 20 & 41 & 89 & 192 & 408 & 869 & 1856 & 3953 \\
$(1<>)$ & 1 & 1 & 3 & 4 & 7 & 12 & 19 & 29 & 48 & 75 \\
$(1 \leqslant \geqslant)$ & 1 & 2 & 4 & 8 & 16 & 28 & 52 & 91 & 161 & 280 \\
$(0 \leqslant>)$ & 4 & 10 & 22 & 51 & 115 & 256 & 571 & 1273 & 2836 & 6315 \\
$(1 \leqslant>)$ & 1 & 2 & 3 & 5 & 8 & 13 & 22 & 36 & 57 & 93 \\
\hline
\end{tabular}

\begin{tabular}{|c|c|c|c|c|c|}
\hline & $(0<>)$ & $(1<>)$ & $(1 \leqslant \geqslant)$ & $(0 \leqslant>)$ & $(1 \leqslant>)$ \\
\hline$A$ & 2.04984811 & 0.82222360 & 1.14429868 & 2.10981051 & 0.84100927 \\
\hline$r$ & 0.469323708 & 0.636281750 & 0.576148769 & 0.449139726 & 0.624235520 \\
\hline$n=5$ exact & 89 & 7 & 16 & 115 & 8 \\
\hline$n=5 \quad A r^{-n}$ & 90 & 8 & 18 & 115 & 9 \\
\hline$n=10$ exact & 3,953 & 75 & 280 & 6,315 & 93 \\
\hline$n=10 A r^{-n}$ & 3,954 & 76 & 284 & 6,316 & 94 \\
\hline$n=20$ exact & $7,625,560$ & 6,949 & 70,438 & $18,906,628$ & 10,419 \\
\hline$n=20 A r^{-n}$ & $7,625,561$ & 6,950 & 70,444 & $18,906,627$ & 10,419 \\
\hline
\end{tabular}

Table 1: The number of alternating compositions for small $n$.

Table 2: A comparison of exact and $A r^{-n}$ estimates for compositions of $n$. 
These lead to

$$
\begin{aligned}
f_{<}(s, t, x, z) & =A(s, t, x, z)+B(s, t, x, z) \frac{A(s, t, x, 1)}{1-B(s, t, x, 1)} \\
f_{>}(s, t, x, z) & =\frac{s t}{1-x z}(1-A(s, t, x, x z)) \\
& +\frac{s t}{1-x z}(1-B(s, t, x, x z)) \frac{A(s, t, x, 1)}{1-B(s, t, x, 1)} .
\end{aligned}
$$

where

$$
\begin{aligned}
A(s, t, x, z)= & \sum_{k=1}^{\infty}(-1)^{k-1}\left(1+\frac{s t}{1-x^{2 k} z}\right) \\
\times & \left(\prod_{i=1}^{k-1} \frac{s t}{1-x^{2 i} z}\right)\left(\prod_{i=1}^{k} \frac{t x^{2 i-1} z}{1-x^{2 i-1} z}\right) \\
B(s, t, x, z)= & \sum_{k=1}^{\infty}(-1)^{k-1} \prod_{i=1}^{k}\left(\frac{s t}{1-x^{2 i} z} \frac{t x^{2 i-1} z}{1-x^{2 i-1} z}\right)
\end{aligned}
$$

The generating function for the number of $(0<>)$-compositions by number of parts and sum of parts is

$$
g(t, x)=f_{>}(1, t, x, 1)+f_{<}(1, t, x, 1)-\frac{t x}{1-x},
$$

where the subtraction occurs because a single nonzero part is counted by both $f_{>}$and $f_{<}$. The generating function for the number of $(1<>)$-compositions is $g(t x, x)$.

\section{References}

[1] E.A. Bender, Central and local limit theorems applied to asymptotic enumeration, J. Combinatorial Theory 15 (1973) 91- 111.

[2] E.A. Bender and E.R. Canfield, Locally restricted compositions I. Restricted adjacent differences, Elec. J. Combin. 12(1) (2005) R57, 27pp.

[3] E.A. Bender and E.R. Canfield, Locally restricted compositions II. General restrictions and infinite matrices. Elec. J. Combin. 16(1) (2009) R108, 35pp. 